\title{
IMPACTO DE LAS METÁSTASIS DEL LINFONODO CENTINELA Y SU TAMAÑO, EN EL TRATAMIENTO DEL CÁNCER DE MAMA*
}

\author{
Drs. Nicolás Escudero M. ${ }^{1}$, Jaime Jans B. ${ }^{1}$, Felipe León F. ${ }^{1}$, Augusto León R. ${ }^{1}$, \\ Francisco Domínguez C. ${ }^{1}$, Ignacio Goñi E. ${ }^{1}$, Nicolás Droppelman M. ${ }^{1}$, \\ César Sánchez R. ${ }^{2}$, David Oddó B. ${ }^{3}$, Mauricio Camus A. ${ }^{1}$ \\ Departamento de Cirugía Oncológica y Maxilofacial. \\ Departamento de Hematología-Oncología. \\ 3 Departamento de Anatomía Patológica. \\ Pontificia Universidad Católica de Chile. \\ Santiago, Chile.
}

Abstract

\section{Impact of sentinel lymph node metastases in the treatment of breast cancer}

Introduction: The importance of sentinel lymph nodes (SL) metastasis at breast cancer patients has been questioned and observation without axillary dissection (AD) associated with adjuvant therapies has been the recommendation in recent years. Objective: To evaluate the macro (MA), micro (MI) and submicrometastasis (SM) of breast cancer in SL, and their impact on the posterior AD. Methods: We reviewed results of biopsies from patients with invasive breast cancer with MA, MI and SM found in the SL operated at our institution between May 1999 and December 2011. Results: We found 134 patients with MA, 33 patients with MI and 30 patients with SM, in a total of 632 patients with invasive breast cancer in those who underwent SL. These were operated 130, 24 and 17 patients respectively. The frequency of no sentinels lymph nodes (NSL) with metastases found on AD was 46.9\% (61/130) for MA, 33.3\% (8/24) for MI and 23.5\% (4/17) for SM. The NSL metastasis from MA modified the TNM in 26.9\% (35/130), those from MI in 20.8\% (5/24) patients, while the SM only modified in one patient (5.9\%). Conclusions: The frequency of lymph nodal involvement in $\mathrm{AD}$ is significantly higher in patients with MA. The number of $\mathrm{AD}$ without clear therapeutic input is high and increases with decreasing size of SL metastases. The results support to not perform AD in patients with MI and SM in the SL, who received conservative surgery and adjuvant therapy.

Key words: Breast cancer, sentinel lymph node biopsy, micrometastases, submicrometastasis, axillary lymph node dissection.

\section{Resumen}

Introducción: La importancia de las metástasis encontradas en linfonodos centinelas (LC) de pacientes con cáncer de mama ha sido cuestionada, por lo que frente al hallazgo de éstas, la observación sin disección

*Recibido el 8 de julio de 2013 y aceptado para publicación el 6 de agosto de 2013.

Los autores declaran no tener conflicto de interés.

Correspondencia: Dr. Mauricio Camus A.

Marcoleta 352, Santiago, Chile. Fax: 5626396395

mcamus@med.puc.cl 
axilar (DA), asociada a terapias adyuvantes, ha sido considerada como una alternativa en los últimos años. Objetivo: Evaluar las macro (MA), micro (MI) y submicrometástasis (SM) de cáncer de mama en LC, y su impacto en la DA posterior. Materiales y Métodos: Se revisaron los resultados de las biopsias de pacientes con cáncer de mama invasor con MA, MI y SM encontradas en el LC operadas en nuestra institución, entre mayo de 1999 y diciembre de 2011. Resultados: Se encontraron 134 pacientes con MA, 33 pacientes con MI y 30 SM, dentro de 632 pacientes con cáncer de mama invasor a los que se les realizó LC. De estos se operaron 130, 24 y 17 pacientes, respectivamente. La frecuencia de Linfonodos No Centinelas (LNC) con metástasis encontradas en la DA fue de 46,9\% (61/130) para MA, 33,3\% (8/24) para MI y 23,5\% (4/17) para SM. Las metástasis del LNC provenientes de MA modificaron el TNM en 26,9\% (35/130), las provenientes de MI en 20,8\% (5/24) pacientes, mientras que las SM sólo lo modificaron en un paciente (5,9\%). Conclusiones: La frecuencia del compromiso linfonodal en la DA es significativamente mayor en las pacientes con MA. El número de DA sin claro aporte terapéutico es alto y aumenta al disminuir el tamaño de las metástasis en el LC. Los resultados apoyan no realizar la DA en pacientes con MI y SM en el LC, que hayan recibido tratamiento quirúrgico conservador y vayan a recibir adyuvancia sistémica.

Palabras clave: Cáncer de mama, linfonodo centinela, micrometástasis, submicrometástasis, disección axilar.

\section{Introducción}

La técnica de biopsia del linfonodo centinela (LC) para cáncer de mama, ha probado ser el estándar para las pacientes en etapas tempranas del cáncer, sin evidencia clínica de metástasis axilar ${ }^{1}$. La disección axilar (DA) representa el tratamiento estándar ante la presencia de metástasis en el LC. El uso de la técnica del LC ha disminuido el número de disecciones axilares y sus complicaciones asociadas $^{2}$, habiendo demostrado ser eficaz para determinar la etapificación del compromiso axilar ${ }^{3}$, siendo este último fundamental para el pronóstico de las pacientes.

Las técnicas histopatológicas han presentado avances que permiten detectar las metástasis en LC de manera más efectiva, gracias a los avances de la biología molecular y la calidad de los equipos utilizados. Dado esto, en los últimos años se ha detectado metástasis cada vez de menor tamaño. La American Joint Committee on Cancer (AJCC) ha clasificado las metástasis en los LC según su tamaño en macrometástasis (MA): mayor de 2,0 $\mathrm{mm}$; micrometástasis (MI): mayor de $0,2 \mathrm{~mm}$ y hasta $2 \mathrm{~mm}$ y submicrometástasis (SM): hasta 0,2 $\mathrm{mm}$ (Tabla 1). La incidencia reportada de MI en LC varía de 15 a 48\%, con una incidencia de metástasis en los linfonodos no centinelas (LNC) de 0 a $57 \%{ }^{4}$. Las recomendaciones de la ASCO del 2005 plantearon la necesidad de realizar cortes seriados e inmunohistoquímica al estudio de los $\mathrm{LC}^{1}$, sin embargo, en la actualidad la recomendación ha sido, la de no realizar técnicas especiales en el estudio del $\mathrm{LC}^{5,6}$.

Existen antecedentes controversiales a nivel mundial ${ }^{7,8}$ respecto del pronóstico de las MI y SM, con recomendaciones poco concluyentes respecto a realizar o no tratamiento a nivel axilar, sea disección o radioterapia, a aquellas pacientes con el hallazgo de MI y SM.

El objetivo de este estudio es determinar el impacto de la presencia de metástasis en LNC en pacientes con MA, MI y SM en los LC.

Los objetivos específicos son:

- Determinar la frecuencia global de metástasis axilares en pacientes con cáncer de mama invasor.

- Determinar la histología de los tumores que generaron las metástasis axilares.

- Determinar la frecuencia de MA, MI, SM en LC.

- Determinar la frecuencia de metástasis en los LNC, en LC con MA, MI y SM.

\section{Materiales y Métodos}

\section{Diseño}

Estudio retrospectivo descriptivo-analítico.

\section{Pacientes}

Se incluyó a todas las pacientes operadas por cáncer de mama invasor entre mayo de 1999 y diciembre de 2011 en el Hospital Clínico de la Pontificia Universidad Católica de Chile, en que se realizó biopsia del LC. Se excluyeron las pacientes con carcinoma microinvasor (T1mic) ya que ninguno de estos presentó metástasis en los linfonodos centinelas. También se excluyeron aquellas pacientes que recibieron neoadyuvancia. La biopsia se realizó con cortes seriados con tinción de Hematoxilina-Eosina y estudio de inmunohistoquímica para pancitoqueratinas. La técnica de biopsia del LC empleada en la mayoría de las pacientes fue la técnica mixta (linfocintigrafía con inyección de Nanoscint ${ }^{\mathbb{B}}$ marcado con Tecnecio ${ }^{99 \mathrm{~m}}$, con uso de sonda intraoperatoria detectora de radiactividad y tinta azul patente al $1 \%)$. 
Tabla 1. Clasificación linfonodal patológica (pN) AJCC 2010

\begin{tabular}{|c|c|}
\hline pNx & $\begin{array}{l}\text { No se pueden evaluar los linfonodos regionales (p. ej., extirpación previa o no se extirpó para un estudio } \\
\text { patológico) }\end{array}$ \\
\hline \multirow[t]{2}{*}{ pNo } & No se identificó metástasis en linfonodos regionales por medios histológicos \\
\hline & $\begin{array}{l}\text { Nota: Las células tumorales aisladas o ITC (de la sigla en inglés isolated tumor cells) se definen como pe- } \\
\text { queños grumos de células } \leq 0,2 \mathrm{~mm} \text {, o células de un sólo tumor o una aglomeración de }<200 \text { células en una } \\
\text { sección histológica cruzada única. Las ITC se pueden localizar mediante una histología rutinaria o mediante } \\
\text { métodos de inmunohistoquímica (IHC). Los nódulos que sólo contienen ITC se excluyen del recuento total } \\
\text { de nódulos positivos para el proceso de clasificación N, pero se deben incluir en el número total de nódulos } \\
\text { evaluados }\end{array}$ \\
\hline pNo(i-) & Histológicamente, no hay metástasis regional a linfonodos, IHC negativo \\
\hline pNO(i+) & Células malignas en linfonodos regionales $\leq 0,2 \mathrm{~mm}$ (detectado mediante $\mathrm{H} \& \mathrm{E}$ o IHC, incluyendo la ITC) \\
\hline pNO(mol-) & Histológicamente no hay metástasis a linfonodos regionales, hallazgos moleculares negativos (RT-PCR) \\
\hline pNo(mol+) & $\begin{array}{l}\text { Hallazgos moleculares positivos (RT-PCR), pero no se localizó metástasis a linfonodos regionales mediante } \\
\text { histología o IHC }\end{array}$ \\
\hline pN1 mi & Micrometástasis $(>0,2 \mathrm{~mm}$ o $>200$ células pero ninguna $>2,0 \mathrm{~mm})$ \\
\hline pN1a & Metástasis en 1-3 linfonodos axilares, al menos una metástasis $>2,0 \mathrm{~mm}$ \\
\hline pN1b & $\begin{array}{l}\text { Metástasis en linfonodos mamarios internos con micrometástasis o macrometástasis detectadas mediante } \\
\text { biopsia de linfonodo centinela, pero sin detección clínica }\end{array}$ \\
\hline pN1c & $\begin{array}{l}\text { Metástasis en 1-3 linfonodos axilares y linfonodos mamarios internos con micrometástasis o macrometástasis } \\
\text { localizadas mediante biopsia, sin detección clínica. }\end{array}$ \\
\hline pN2a & Metástasis en 4-9 linfonodos axilares (al menos un depósito tumoral > 2 mm) \\
\hline $\mathbf{p N 2 b}$ & $\begin{array}{l}\text { Metástasis en linfonodos mamarios internos detectados clínicamente en ausencia de metástasis a linfonodo } \\
\text { axilar }\end{array}$ \\
\hline pN3a & $\begin{array}{l}\text { Metástasis en } \geq 10 \text { linfonodos axilares (por lo menos un depósito tumoral }>2,0 \mathrm{~mm} \text { ) o metástasis a los lin- } \\
\text { fonodos infraclaviculares }\end{array}$ \\
\hline pN3b & $\begin{array}{l}\text { Metástasis en }>3 \text { linfonodos axilares y linfonodos mamarios internos detectados clínicamente en presencia } \\
\text { de metástasis a linfonodo axilar; o metástasis en }>3 \text { linfonodos axilares y linfonodos mamarios internos con } \\
\text { micrometástasis o macrometástasis localizada mediante biopsia de linfonodo centinela, sin detección clínica }\end{array}$ \\
\hline pN3c & Metástasis en linfonodos ipsilaterales supraclaviculares \\
\hline
\end{tabular}

\section{Fuentes}

Registro prospectivo de fichas clínicas y biopsias del Departamento de Anatomía Patológica del Hospital Clínico de la Pontificia Universidad Católica de Chile.

\section{Variables}

Se consideraron: Edad, tamaño tumoral, resultados de anatomía patológica, resultados de biopsia diferida del LC, presencia de MA, MI y SM. Se consideró MA aquellas mayores de $2 \mathrm{~mm}$, MI aquellas mayores de $0,2 \mathrm{~mm}$ y hasta $2 \mathrm{~mm}$ y SM aquellas de hasta $0,2 \mathrm{~mm}$.

\section{Análisis estadístico}

Para el análisis estadístico se utilizó el programa Statcalc V.6. Se realizó estadística descriptiva. Para comparar pruebas, se realizó pruebas de hipótesis paramétricas y no paramétricas según la determinación de normalidad. Se consideró estadísticamente significativo un nivel de confianza del $95 \%$ con un valor $\mathrm{p}<0,05$.

\section{Resultados}

Se realizó biopsia de LC a 717 pacientes durante el período analizado. Treinta y dos pacientes correspondieron a carcinomas in situ, 34 a carcinomas microinvasores y 651 correspondieron a cáncer de mama invasor, de los cuales 19 recibieron neoadyuvancia, siendo 632 pacientes el grupo analizado. La mediana de edad de las pacientes fue 55 años (rango de 29 a 89 años).

Se detectaron metástasis en LC en 197/632 casos $(31,2 \%)$, aumentado su frecuencia en relación di- 
recta al tamaño tumoral encontrado, siendo $23,6 \%$ en tumores de hasta $2 \mathrm{~cm}$ y $46,3 \%$ en tumores mayores de $2 \mathrm{~cm}(\mathrm{p}<0,0001)$. De los 197 pacientes con metástasis en el LC, 134 correspondieron a MA (68\%), 33 a MI $(16,8 \%)$ y 30 a SM $(15,2 \%)$. La tinción con hematoxilina eosina reconoció 30 de las 33 MI y 14 de 30 SM. Al comparar los tumores de hasta $2 \mathrm{~cm}$ y los mayores de $2 \mathrm{~cm}$, no encontramos diferencias significativas en la frecuencia de MA $(64,3 \%$ vs $71,7 \%$; $\mathrm{p}=0,265)$, MI $(18,4 \%$ vs $15,2 \% ; \mathrm{p}=0,548)$ ni SM (17,3\% vs $12,1 \%$; p = 0,302). La frecuencia y tipo de metástasis en el LC se muestra en la Tabla 2.

La histología más frecuente fue el adenocarcinoma ductal, representando el $82 \%$ de la serie, con un $32 \%$ de LC metastásicos. Al comparar la frecuencia de LC metastásicos con la histología lobulillar no encontramos diferencia estadística significativa (32\% vs $40 \%$, respectivamente; $p=0,27)$. La frecuencia de LC metastásicos de los adenocarcinomas tubulares fue significativamente menor (32\% vs 4,3\%, respectivamente; $p$ $=0,005)$. El bajo número de casos en las otras histologías invalidó la comparación (Tabla 3).

Ciento setenta y un casos $(86,8 \%)$ fueron tratados con DA, encontrándose metástasis adicionales en los LNC en 73 casos (42,7\%). Se realizó disección axilar en 130 pacientes con MA (97\%), 24 con MI (72,7\%) y 17 con SM $(56,7 \%)$. La frecuencia de metástasis en los LNC fue de 46,9\% en MA (61/130), $33,3 \%$ en MI (8/24), y $23,5 \%$ en SM (4/17). Tabla 4.

Las metástasis encontradas en LNC provenientes de MA cambiaron la clasificación linfonodal en 35/130 casos $(26,9 \%)$ de las cuales 27 pasaron de pN1a a pN2 y 8 a pN3. De los casos con MI, 5/24 (20,8\%) modificaron su clasificación linfonodal, de los cuales 3 pasaron de $\mathrm{pN} 1 \mathrm{mic}$ a $\mathrm{pN} 1 \mathrm{a}$, y 2 a pN2. Sólo 1/17 (5,9\%) casos de SM modificó su clasificación linfonodal de pN0 (i+) a pN1. Al comparar los porcentajes de modificación no encontramos diferencia estadísticamente significativa entre las MA, MI ni $\mathrm{SM}(\mathrm{p}=0,149)$. Al correlacionar el tamaño de las metástasis linfonodales y el tamaño tumoral, encontramos que los tumores de hasta $2 \mathrm{~cm}$ tuvieron una menor frecuencia de cambio en la etapa linfonodal, sin embargo, esta diferencia no fue significativa para las MA, MI ni SM (Tabla 5).
Tabla 2. Número de pacientes con linfonodo centinela positivo, según estadio tumoral $(T)$ y estratificado por tamaño de la metástasis en el LC

\begin{tabular}{|c|c|c|c|c|c|c|}
\hline \multirow{2}{*}{$\begin{array}{l}\text { Tamaño } \\
\text { (T) }\end{array}$} & \multirow[b]{2}{*}{$\mathbf{n}$} & \multicolumn{5}{|c|}{ Linfonodo centinela positivo en biopsia diferida } \\
\hline & & MA & MI & SM & Total & $\% \mathrm{LC}+$ \\
\hline $\mathrm{T} 1$ & 416 & 63 & 18 & 17 & 98 & 23,6 \\
\hline T1a & 36 & 3 & 2 & 0 & 5 & 16,7 \\
\hline $\mathrm{T} 1 \mathrm{~b}$ & 110 & 10 & 3 & 5 & 18 & 16,4 \\
\hline T1c & 270 & 50 & 13 & 12 & 75 & 27,8 \\
\hline $\mathrm{T} 2$ & 195 & 59 & 15 & 12 & 86 & 44,1 \\
\hline $\mathrm{T} 3$ & 17 & 10 & 0 & 1 & 11 & 64,7 \\
\hline $\mathrm{T} 4$ & 2 & 2 & 0 & 0 & 2 & 100,0 \\
\hline Sin certeza & 2 & 0 & 0 & 0 & 0 & 0,0 \\
\hline Total & 632 & 134 & 33 & 30 & 197 & 31,2 \\
\hline
\end{tabular}

$\mathrm{MA}=$ Macrometástasis, $\mathrm{MI}=$ Micrometástasis, $\mathrm{SM}=$ Submicrometástasis.

Tabla 3. Compromiso de LC según histología

\begin{tabular}{|lrrrrc|}
\hline Histología & $\begin{array}{c}\mathbf{n} \\
\text { total }\end{array}$ & $\mathbf{\%}$ & \multicolumn{2}{c}{ Casos con LC+ } & $\mathbf{p}^{\wedge}$ \\
Ductal & 518 & 82,0 & 166 & 32,0 & $\ldots$ \\
\hline Lobulillar & 45 & 7,1 & 18 & 40,0 & 0,27 \\
Mixto & 13 & 2,1 & 5 & 38,5 & $*$ \\
Papilar & 12 & 1,9 & 4 & 33,3 & $*$ \\
Medular & 8 & 1,3 & 2 & 25,0 & $*$ \\
Mucinoso & 11 & 1,7 & 1 & 9,1 & $*$ \\
Tubular & 23 & 3,6 & 1 & 4,3 & 0,005 \\
Adenoideo quístico & 2 & 0,3 & 0 & 0,0 & $*$ \\
Total & 632 & 100,0 & 197 & 31,2 & \\
\hline
\end{tabular}

${ }^{\wedge}$ Se utilizó la prueba $\chi^{2}$ para calcular la asociación entre la proporción de LC+ entre la histología ductal y el resto de las histologías. * La significación estadística de la prueba $\chi^{2}$ no es válida ya que el $25 \%$ de los resultados esperados son menores de 5 .

Tabla 4. Porcentaje de pacientes tratados con disección axilar y frecuencia de LNC metastásicos según tamaño de metástasis en el LC

\begin{tabular}{|crrrrrr|}
\hline $\begin{array}{c}\text { Metástasis en el } \\
\text { LC }\end{array}$ & \multicolumn{4}{c}{$\begin{array}{c}\text { Pacientes con } \\
\text { DA }\end{array}$} & \multicolumn{4}{c|}{$\begin{array}{c}\text { Pacientes } \\
\text { LNC + }\end{array}$} \\
Tipo & n & n & $\mathbf{\%}$ & \multicolumn{1}{c|}{ n } & \% & $p$ \\
MA & 134 & 130 & 97,0 & 61 & 46,9 & $\ldots$ \\
MI & 33 & 24 & 72,7 & 8 & 33,3 & 0,218 \\
SM & 30 & 17 & 56,7 & 4 & 23,5 & 0,067 \\
MI y SM & 63 & 41 & 65,1 & 12 & 29,3 & 0,047 \\
Total & 197 & 171 & 86,8 & 73 & 42,7 & \\
\hline
\end{tabular}

$\mathrm{MA}=$ Macrometástasis, $\mathrm{MI}=$ Micrometástasis, $\mathrm{SM}=$ Submicrometástasis 
Tabla 5. Modificación en la etapa N, post disección axilar en pacientes con Macrometástasis (MA), Micrometástasis (MI) y Submicrometástasis (SM) en LC, según tamaño tumoral

\begin{tabular}{|c|c|c|c|c|c|c|c|c|c|}
\hline $\begin{array}{l}\text { Etapa N } \\
\text { pre DA }\end{array}$ & Con DA & LNC+ & pNo (i+) & $\begin{array}{c}\text { Eta } \\
\text { pNomic }\end{array}$ & $\begin{array}{l}\text { N post } \\
\text { pN1a }\end{array}$ & pN2 & pN3 & Modificación\% & $\mathbf{p}$ \\
\hline MA pN1a & 130 & 61 & 0 & 0 & 26 & 27 & 8 & 26,9 & --- \\
\hline $\mathrm{Tu} \leq 2 \mathrm{~cm}$ & 61 & 30 & 0 & 0 & 17 & 9 & 4 & 21,3 & \\
\hline $\mathrm{Tu}>2 \mathrm{~cm}$ & 69 & 31 & 0 & 0 & 9 & 18 & 4 & 31,9 & 0,17 \\
\hline MI pN1mic & 24 & 8 & 0 & 3 & 3 & 2 & 0 & 20,8 & $0,53 *$ \\
\hline $\mathrm{Tu} \leq 2 \mathrm{~cm}$ & 14 & 3 & 0 & 1 & 1 & 1 & 0 & 14,3 & \\
\hline $\mathrm{Tu}>2 \mathrm{~cm}$ & 10 & 5 & 0 & 2 & 2 & 1 & 0 & 30,0 & \\
\hline SM pNO(i+) & 17 & 4 & 3 & 0 & 1 & 0 & 0 & 5,9 & $0,058 *$ \\
\hline $\mathrm{Tu} \leq 2 \mathrm{~cm}$ & 11 & 2 & 2 & 0 & 0 & 0 & 0 & 0,0 & \\
\hline $\mathrm{Tu}>2 \mathrm{~cm}$ & 6 & 2 & 1 & 0 & 1 & 0 & 0 & 16,7 & \\
\hline
\end{tabular}

MA= Macrometástasis, $\mathrm{MI}=$ Micrometástasis, $\mathrm{SM}=$ Submicrometástasis, $\mathrm{DA}=$ Disección Axilar, $\mathrm{LNC}=$ Linfonodo No Centinela *= se comparó contra macrometástasis, con prueba de $\chi^{2}$.

\section{Discusión}

El LC representa la técnica estándar en la etapificación axilar del cáncer de mama y su mejor rendimiento en la detección de metástasis nos ha llevado a enfrentarnos a la problemática de pacientes que presentan el hallazgo de $\mathrm{MI}$ o $\mathrm{SM}^{6,8,9}$. En nuestro centro, el $31,2 \%$ de las pacientes con cáncer de mama presentaron metástasis en el LC, siendo mayor la proporción de compromiso, a medida que aumenta el tamaño tumoral. Dado que la gran mayoría de los casos correspondían a tumores T1c y T2, los agrupamos en tumores de hasta $2 \mathrm{~cm}$ y mayores de $2 \mathrm{~cm}$, encontrando diferencias significativas en la frecuencia de compromiso de LC. Las MI y SM representaron el $32 \%$ de estas metástasis, concordante con otros reportes ${ }^{4}$, sin diferencias significativas en relación al tamaño tumoral.

El American College of Surgeons Oncology Group (ACOSOG), en su protocolo randomizado Z0011 $1^{5}$, evaluaron el efecto en la sobrevida, de completar la disección axilar en pacientes con metástasis en el LN, sin encontrar diferencias entre las pacientes sin y con disección axilar, en sobrevida global $(91,8 \%$ vs $92,5 \% ; \mathrm{p}=0,25)$ ni en la sobrevida libre de enfermedad $(82,2 \%$ vs $83,9 \% ; p=0,14)$ a 5 años. Todas las pacientes recibieron tratamiento conservador a la mama y casi todas recibieron tratamiento sistémico adyuvante (96\% y 97\%, respectivamente). Si bien el estudio no logró reclutar el número planificado de pacientes, sus resultados motivaron un cambio de conducta a nivel mundial. En nuestra serie, no realizamos la disección axilar en el 3\% de pacientes con MA en el LC, el 27,3\% de pacientes con MI y el $43,3 \%$ de pacientes con SM, tomando en cuenta variables como la edad, comorbilidades, el uso de terapias adyuvantes y la decisión consensuada en comité oncológico.

La frecuencia de MA en el LC fue mayor que la presencia de MI y SM juntas (68\% vs 32\%). Es importante destacar que 30 de las $33 \mathrm{MI}$ fueron detectadas con H-E, es decir, sólo 3 casos fueron detectados sólo por técnicas de inmunohistoquímica. En el caso de las SM, 14 de 30 casos fueron detectados por H-E y sólo 16 casos por técnicas de inmunohistoquímica. La dudosa relevancia de las SM y la alta capacidad de detección de MI con técnica de H-E, apoya la conducta de discontinuar el uso de técnicas de inmunohistoquímicas para su detección $n^{5,6}$.

La presencia de MA se asoció a una mayor frecuencia de LNC comprometidos que las MI y SM juntas $(46,9 \%$ vs $29,3 \%$; $p=0,047)$, concordante con otras series ${ }^{10,11}$. El cambio de etapificación linfonodal secundario a LNC metastásicos fue de 26,9\% para MA. Para MI y SM fue de $20,8 \%$ y $5,9 \%$; respectivamente (Tabla 5). Dicho de otra forma, lo más importante de esto pareciera ser el hecho de que el $73,1 \%, 79,2 \%$ y $94,1 \%$ de las DA, no modifican la estadificación y por ende no ofrecen un claro beneficio en el tratamiento de pacientes con MA, MI y $\mathrm{SM}$, respectivamente.

Varios autores han evaluado el pronóstico de las MI y SM y la relevancia de $\mathrm{DA}^{4,12}$. Bilimoria et $\mathrm{al}^{13}$, en un análisis retrospectivo de la Base de datos de Cáncer de USA, compararon los resultados de los pacientes con LC metastásico, tratados sin y con DA, sin encontrar diferencias en los pacientes con MI (recurrencia axilar: $0,6 \%$ vs $0,2 \% ; p=0,063$; sobrevida a 5 años: $88,6 \%$ vs $90,9 \% ; \mathrm{p}=0,16$ ) ni tampoco en los pacientes con MA (recurrencia axilar: $1,2 \%$ vs $1,0 \% ; \mathrm{p}=0,4$; sobrevida a 5 años: $81,3 \%$ vs $81,8 \% ; p=0,63)$, observando que la di- 
sección axilar no agrega beneficio a los resultados. Langer et $\mathrm{al}^{14}$, en un estudio prospectivo comparó los resultados de pacientes con LC sin metástasis y con MI, a los que no se les realizó DA, no encontraron diferencias en sobrevida global $(93,1 \%$ vs $92,6 \% ; p=0,656)$, sobrevida libre de enfermedad $(90,7 \%$ vs $100 \% ; p=0,081)$ ni recurrencia locoregional $(94,1 \%$ vs $100 \% ; p=0,174)$ a 5 años. El $96,3 \%$ de las pacientes con MI recibieron algún tipo de terapia sistémica (hormonoterapia y/o quimioterapia) comparado con un $86,4 \%$ en el grupo con LC negativo $(\mathrm{p}=0,091)$. De Boer et $\mathrm{a}^{15}$, en un estudio retrospectivo, analizaron los resultados de pacientes sin metástasis en el LC tratados sin terapia sistémica adyuvante (grupo 1), pacientes con LC con MI o células tumorales aisladas tratadas sin terapia sistémica (grupo 2) y pacientes con LC con MI o células tumorales aisladas tratadas con terapia sistémica (grupo 3). El 14\% de los pacientes en el grupo 1 recibió tratamiento a la axila con DA o radioterapia, comparado con el $54,6 \%$ en el grupo 2 y el $75,4 \%$ en el grupo $3(p<0,001)$. Encontraron que los pacientes con MI tratados sin terapia sistémica tuvieron menor sobrevida libre de enfermedad a 5 años que los pacientes sin metástasis en el LC $(76,5 \%$ vs $85,7 \% ; p<0,001)$. Los pacientes con MI tratados con terapia sistémica mejoraban su sobrevida libre de enfermedad a 5 años en comparación a los que no la recibían $(86,2 \%$ vs $76,5 \%$; $<<0,001)$ siendo similar al grupo de pacientes sin metástasis en el LC, lo que destaca la importancia de las terapias adyuvantes. Hansen et $\mathrm{al}^{16}$, en un estudio propectivo evaluaron el impacto en sobrevida de las MI en los LC; encontraron que los pacientes con MI no tenían peor pronóstico que los pacientes sin compromiso metastásico axilar (sobrevida libre de enfermedad: $92,1 \%$ vs $95,9 \% ; \mathrm{p}=$ no especificado; sobrevida global: $94,1 \%$ vs $96,9 \%$; $\mathrm{p}=$ no especificado) a 8 años, sin embargo, en los pacientes con MI hubo más DA ( $87 \%$ vs $32 \%)$ y se usó más frecuentemente tratamiento sistémico $(96,3 \%$ vs $66,1 \%)$. Por último, Galimberti et $\mathrm{al}^{17}$, en un estudio randomizado, analizaron los resultados a 5 años, de pacientes con MI en el LC, tratados sin y con DA; no hubo diferencias significativas en sobrevida libre de enfermedad $(87,5 \%$ vs $84,4 \%: \mathrm{p}=0,16)$ ni sobrevida global $(97,5 \%$ vs $97,6 \%: p=0,73)$. Se encontró sólo un $13 \%$ de LNC en la DA. El 91\% de ambos grupos recibió tratamiento quirúrgico conservador y sobre el $95 \%$ de ambos grupos recibieron terapia sistémica.

Recientemente hemos reportado el rendimiento diagnóstico de la biopsia rápida del LC, sugiriendo la no realización de ésta en pacientes con tumores de hasta $2 \mathrm{~cm}$. Esto por la baja frecuencia de LC metastásico en tumores de hasta $2 \mathrm{~cm}$ y el alto porcentaje de falsos negativos de la técnica de biopsia rápida $(33 \%)$ para la detección de metástasis ${ }^{18}$. Los resultados del presente reporte hacen plantear la posibilidad de discontinuar el uso de biopsia rápida independiente del tamaño tumoral, esto debido al alto número de DA con dudoso beneficio, lo que orienta a evaluar la decisión de DA en equipos multidisciplinarios, teniendo en cuenta, el reporte de Straver et $\mathrm{al}^{19}$, del estudio AMAROS, en el que el conocimiento del estado axilar post disección no modificó la administración de tratamiento adyuvante.

Creemos que por el momento la omisión de la disección axilar debe ser considerada basándose en los criterios del estudio Z0011 (tumores T1-T2, invasores, sin adenopatías palpables, con 1 ó 2 LC metastásicos), a la espera de los resultados de seguimiento de otros estudios como el AMAROS. En pacientes con MI y SM en el LC, la menor frecuencia de LNC comprometidos y la baja frecuencia de cambio en la etapa linfonodal orienta a no realizar la disección axilar, teniendo presente que los datos existentes en relación al pronóstico, apoyan esta conducta en pacientes con tratamiento quirúrgico conservador y adyuvancia con hormonoterapia y/o quimioterapia. $\mathrm{La}$ incertidumbre actual acerca del real pronóstico de las MI y SM, apoya la conducta propuesta en St. Gallen 2011 de no usar técnicas de alta sensibilidad en su detección ${ }^{6}$.

\section{Conclusiones}

Un tercio de los pacientes con cáncer de mama invasor en nuestra serie, presentan compromiso metastásico en el LC. Las MI y SM representan un tercio de las metástasis linfonodales. La frecuencia del compromiso linfonodal en la disección axilar es significativamente mayor en las pacientes con MA. La frecuencia de modificación en la etapa $\mathrm{N}$ también fue mayor para MA, sin embargo, no alcanzó significación estadística, probablemente debido al número de casos comparados. El número de DA sin claro aporte terapéutico es alto y aumenta al disminuir el tamaño de las metástasis linfonodales. Los resultados apoyan no realizar la disección axilar en pacientes con MI y SM en el LC, que hayan recibido tratamiento quirúrgico conservador y vayan a recibir adyuvancia sistémica. La no disección axilar en pacientes con MA debe aún ser evaluada con cautela y discutida en equipos multidisciplinarios.

\section{Referencias}

1. Lyman GH, Giuliano AE, Somerfield MR, Benson AB 3rd, Bodurka DC, Burstein HJ, et al. American Society of Clinical Oncology guideline recommendations for 
sentinel lymph node biopsy in early-stage breast cancer. J Clin Oncol. 2005;23:7703-20.

2. Samphao S, Eremin JM, El-Sheemy M, Eremin O. Management of the axilla in women with breast cancer: Current clinical practice and a new selective targeted approach. Ann Surg Oncol. 2008;15:1282-96.

3. Veronesi U, Galimberti V, Paganelli G, Maisonneuve P, Viale G, Orecchia R, et al. Axillary metastases in breast cancer patients with negative sentinel nodes: a followup of 3548 cases. Eur J Cancer 2009;45:1381-8.

4. Salhab M, Patani N, Mokbel K. Sentinel lymph node micrometastasis in human breast cancer: an update. Surg Oncol. 2011;20:e195-206.

5. Giuliano AE, Hunt KK, Ballman KV, Beitsch PD, Whitworth PW, Blumencranz PW, et al. Axillary dissection vs no axillary dissection in women with invasive breast cancer and sentinel node metastasis: a randomized clinical trial. JAMA 2011;305:569-75.

6. Goldhirsch A, Wood WC, Coates AS, Gelber RD, Thürlimann B, Senn HJ, et al. Strategies for subtypes-dealing with the diversity of breast cancer: highlights of the St. Gallen International Expert Consensus on the Primary Therapy of Early Breast Cancer 2011. Ann Oncol. 2011;22:1736-47.

7. Cote RJ, Peterson HF, Chaiwun B, Gelber RD, Goldhirsch A, Castiglione-Gertsch M, et al. Role of immunohistochemical detection of lymph-node metastases in management of breast cancer. International Breast Cancer Study Group. Lancet. 1999;354(9182):896-900.

8. Dowlatshahi K, Fan M, Anderson JM, Bloom KJ. Occult metastases in sentinel nodes of 200 patients with operable breast cancer. Ann Surg Oncol. 2001;8:675-81.

9. Chen SL, Hoehne FM, Giuliano AE. The prognostic significance of micrometastases in breast cancer: a SEER population-based analysis. Ann Surg Oncol. 2007;14:3378-84.

10. Rutledge H, Davis J, Chiu R, Cibull M, Brill Y, McGrath $\mathrm{P}$, et al. Sentinel node micrometastasis in breast carcinoma may not be an indication for complete axillary dissection. Mod Pathol. 2005;18:762-8.

11. Straver ME, Meijnen $P$, van Tienhoven G, van de Velde
CJ, Mansel RE, Bogaerts J, et al. Sentinel node identification rate and nodal involvement in the EORTC 1098122023 AMAROS trial. Ann Surg Oncol. 2010;17:185461 .

12. De Boer M, van Dijck JA, Bult P, Borm GF, Tjan-Heijnen VC. Breast cancer prognosis and occult lymph node metastases, isolated tumor cells, and micrometastases. J Natl Cancer Inst. 2010;102:410-25.

13. Bilimoria KY, Bentrem DJ, Hansen NM, Bethke KP, Rademaker AW, Ko CY, et al. Comparison of sentinel lymph node biopsy alone and completion axillary lymph node dissection for node-positive breast cancer. J Clin Oncol. 2009;27:2946-53.

14. Langer I, Guller U, Viehl CT, Moch H, Wight E, Harder F, et al. Axillary lymph node dissection for sentinel lymph node micrometastases may be safely omitted in early-stage breast cancer patients: long-term outcomes of a prospective study. Ann Surg Oncol. 2009;16:336674.

15. De Boer M, van Deurzen CH, van Dijck JA, Borm GF, van Diest PJ, Adang EM, et al. Micrometastases or isolated tumor cells and the outcome of breast cancer. N Engl J Med. 2009;361:653-63.

16. Hansen NM, Grube B, Ye X, Turner RR, Brenner RJ, Sim MS, et al. Impact of micrometastases in the sentinel node of patients with invasive breast cancer. J Clin Oncol. 2009;27:4679-84.

17. Galimberti V, Cole BF, Zurrida S, Viale G, Luini A, Veronesi $\mathrm{P}$, et al. Axillary dissection versus no axillary dissection in patients with sentinel-node micrometastases (IBCSG 23-01): a phase 3 randomised controlled trial. Lancet Oncol. 2013;14:297-305.

18. Jans J, Escudero N, León F, León A, Domínguez F, Goñi I, et al. Rendimiento de la biopsia intraoperatoria por congelación en el estudio del linfonodo centinela en cáncer de mama. Rev Chil Cir. 2013;65:115-20.

19. Straver ME, Meijnen $P$, van Tienhoven G, van de Velde CJ, Mansel RE, Bogaerts J, et al. Role of axillary clearance after a tumor-positive sentinel node in the administration of adjuvant therapy in early breast cancer. J Clin Oncol. 2010;28:731-7. 